\title{
MiR-103a targeting Piezo1 is involved in acute myocardial infarction through regulating endothelium function
}

\author{
Lingzhi Huang ${ }^{1,2}$, Lezhi $\mathrm{Li}^{3}$, Xiaofang Chen ${ }^{4}$, Huiling Zhang ${ }^{3}$, Zeya Shi ${ }^{5}$
}

${ }^{1}$ Nursing School, Central South University, Changsha, China

${ }^{2}$ Department of Cardiology, Second Xiangya Hospital, Central South University, Changsha, China

${ }^{3}$ Department of Nursing, Second Xiangya Hospital, Central South University, Changsha, China

${ }^{4}$ Department of Nursing, Third Affiliate Hospital, Southern Medical University, Guangzhou, China

${ }^{5}$ Intesive Care Unit, Hunan Provincial People Hospital, Changsha, China

\begin{abstract}
Background: Acute myocardial infarction (AMI) is commonly known as the heart attack. The molecular events involved in the development of AMI remain unclear. This study was to investigate the expression of miR-103a in patients with high blood pressure (HBP) and AMI patients with and without HBP, as well as its effect on endothelial cell functions.

Methods: MiR-103a expression in plasma and peripheral blood mononuclear cells (PBMCs) was measured by real-time polymerase chain reaction (PCR). The regulatory effect of miR-103a on Piezo1 gene was identified by a luciferase reporter system. The role of miR-103a in endothelial cells was evaluated by the capillary tube formation ability and cell viability of human umbilical vein endothelial cells (HUVECs).
\end{abstract}

Results: The plasma miR-103a concentration was significantly elevated in patients with $H B P$ alone, AMI alone, and comorbidity of AMI and HBP. The miR-103a expression in PBMCs in patients with $A M I$ and HBP was significantly higher than the one in healthy controls $(p<0.05)$, however miR-103a expression in PBMCs was not significantly different among patients with HBP alone, patients with AMI alone, and healthy controls. MiR-103a targeted Piezo1 and inhibited Piezo1 protein expression, which subsequently reduced capillary tube formation ability and cell viability of HUVECs.

Conclusions: MiR-103a might be a potential biomarker of myocardium infarction and could be used as an index for the diagnosis of AMI. It may be involved in the development of HBP and onset of AMI through regulating the Piezo1 expression. (Cardiol J 2016; 23, 5: 556-562)

Key words: acute myocardial infarction, high blood pressure, endothelial cells, miR-103a, Piezo1

Address for correspondence: Dr Lezhi Li, Department of Nursing, Second Xiangya Hospital, Central South University, 139 Renmin Road, Changsha, Hunan 410011, China, tel: +86 13807318762, e-mail: 1126511@sina.com 


\section{Introduction}

Myocardial infarction, commonly known as the heart attack, is a leading cause of mortality in many industrialized countries [1]. Acute myocardial infarction (AMI) occurs when one of the arteries that supply the myocardium is blocked due to acute clot formation. The blockage results in necrosis of myocardial tissue and loss of myocardium function [2]. However, the molecular events involved in the development of AMI are still unclear. Identification of molecular markers for AMI may uncover important triggers involved in the progression of atherosclerotic vascular diseases. Moreover, if a marker can be used to give an early and accurate diagnosis of AMI, it will allow immediate initiation of therapy and reduce the mortality rate.

MicroRNAs (miRNAs) are a family of endogenous, short (17-25 nucleotides) non-coding RNAs. MiRNAs are commonly thought to regulate gene expression at the post-transcriptional level through binding to the 3'-untranslated regions of the mRNA and subsequently inhibiting its translation or inducing its degradation [3]. Bioinformatic analysis predicted that more than $60 \%$ of human genes are targets of miRNAs [4]. Moreover, the accumulated evidence has demonstrated that over 100 circulating miRNAs can be detected in healthy individuals [5]. Although the exact origins and biological functions of circulating miRNAs are still unclear, many studies revealed that circulating miRNAs could be markers of disease progression, prognosis, or diagnosis.

Several miRNAs have been shown to be associated with the pathophysiological consequences of AMI [6]. For example, a study by Long et al. [7] demonstrated that plasma concentration of miR-30a, miR-195, and let-7b are potential indicators of AMI. Other study, by Wang et al. [8], showed that plasma miR-21-5p and miR-361-5p expressions were significantly increased, whereas miR-519e-5p expression was reduced in AMI patients. However, the identification of circulating miRNA in AMI yielded inconsistent findings.

A recent study first identified miR-103a as a mechanosensitive miRNA that regulates osteoblast differentiation by directly targeting Runx2 [9]. The Piezo1 gene has recently been found to be important for normal shear stress-evoked $\mathrm{Ca}^{2+}$ signaling and non-selective cationic channel activity in endothelial cells. Piezo1 channels can function as pivotal integrators in vascular biology [10, 11]. In addition, bioinformatic analysis predicts that Piezo1 is a target of miR-103a, but this prediction has not been validated yet, particularly, the role of miR-103a in AMI. We therefore hypothesize that miR-103a may be involved in cardiovascular diseases through regulating Piezo1 expression in endothelium.

In this study, the circulating miR-103a concentration was compared between patients with high blood pressure (HBP), AMI, and comorbidity of AMI and HBP. The relationship between miR$-103 \mathrm{a}$ and Piezo1, as well as their biological activity, were identified in human umbilical vein endothelial cells (HUVEC).

\section{Methods}

\section{Participants}

Forty-five patients with HBP, 81 patients with AMI (including 38 patients without a history of HBP), and 47 age-matched healthy controls were recruited at Department of Cardiology, Second Xiangya Hospital, Central South University from September 2013 to December 2014. Routine examinations, such as blood glucose, lipid profile, homeostasis, blood pressure, and electrocardiography were performed in each AMI patient immediately after admission. Routine examination in HBP patients included blood glucose, lipid profile, homeostasis, and blood pressure tests. AMI was diagnosed according to published standards [12]. Briefly, the inclusion diagnosis criteria were the following: 1) acute ischemic-type chest pain within $24 \mathrm{~h}$; 2) positive electrocardiogram changes suggesting AMI (new ST-T changes, new left bundle branch block, or new pathological Q-wave changes); and 3) elevated plasma cardiac troponin I ( $>0.1 \mathrm{ng} / \mathrm{mL})$. HBP was determined by 3 examinations at the same time in 3 consecutive days. The blood pressure data of AMI patients were obtained from patient medical records and the most recent record was adopted. All patients with a history of atrial fibrillation, myocardial infarction, hemorrhagic stroke, or recurrent stroke and comorbidity with tumor, acute infectious disease, blood disease, and renal or liver failure were excluded from the study. This study was pre-approved by the Ethics Committee for Human Research, Central South University, and written consent forms were obtained from all participants.

\section{Blood sample collection and RNA extractions}

In AMI patients, blood was collected immediately at admission. In HBP patients and healthy controls, fasting blood was collected. A sample of $10 \mathrm{~mL}$ of whole blood was collected into tubes with 
EDTA anticoagulant, and plasma was isolated from the blood by centrifugation at $1,000 \mathrm{~g}$ for $10 \mathrm{~min}$ at room temperature. At the same time, peripheral blood mononuclear cells (PBMCs) were isolated using Ficoll-400 [13]. Total RNA in plasma and PBMCs was isolated using TRIzol reagent (Life Technologies, Carlsbad, CA, USA) according to the manufacture protocol. The concentration of total RNA was measured using a NanoDrop 1000 spectrophotometer (Thermo Scientific, Utah, USA).

\section{Real-time quantitative PCR}

Reverse transcription was performed using TaqMan miRNA RT Kit (Applied Biosystems, Foster City, USA) by following the manufacturer's protocol. The expression of miRNA-103a was analyzed by real-time (RT) polymerase chain reaction (PCR) using a TaqMan ${ }^{\circledR}$ miRNA assay kit (Life Technologies, USA). The primers for amplifying miR-103a were designed, as previously described [14]. The amplification of each sample was triplicated. The expression of miRNAs was analyzed using the $2^{-\Delta \Delta C t}$ method. Spiked-in caenorhabditis elegans miRNA (cel-miR-39) was used as an internal control for quantitative RT-PCR, as previously described [15]. The relative changes in folds compared to the basal expression were used for data analysis.

\section{Cell culture}

Human umbilical vein endothelial cells (HUVECs) and 293T cell lines were provided by the Shanghai Institute of Cell Biology (China) and cultured in DMEM/F12 medium containing $10 \%$ fetal bovine serum at $37^{\circ} \mathrm{C}, 5 \% \mathrm{CO}_{2}$.

\section{Luciferase reporter assay}

Human Piezo1 cDNA 3'-UTR (NM_001142864) containing the putative binding site of miR-103a was amplified by PCR from a cDNA library prepared from human tissues. The 273bp fragment was amplified using forward primer: 5'-GAGTAGGAGCTGCTGCTGGC-3' and reverse primer: 5'-TCTGATTGTCCATTTGTATAAAT-3' and inserted into the Spe I and Hind III sites of luciferase reporter vector pmiR-REPORT ${ }^{\mathrm{TM}}$ (Life Technologies, USA). The insertion was sequenced using primers provided with the reporter vector. The produced reporting plasmid was named pmiR-Piezo1. 293T cells $\left(1 \times 10^{6}\right)$ were plated in $10 \mathrm{~cm}$ dishes $24 \mathrm{~h}$ before transfection. The commercially synthesized miR-103a (Life Technologies, USA) was co-transfected with pmiR-Piezo1 plasmid DNA or pmiR- REPORT ${ }^{\text {TM }}$ blank plasmid DNA (1:1 mixture) using Lipofectamine 2000 (Life Technolo- gies). Twenty-four hours later, luciferase activities were consecutively measured using a Luciferase 1000 Assay System (Promega, USA) according to the user manual.

\section{Western blot of Piezo1 protein expression}

HUVECs in $10-\mathrm{cm}$ dishes were grown to $60 \%$ confluency and then transfected with $10 \mathrm{mM}$ of miR-103a or $10 \mathrm{mM}$ of cel-miR-39 using lipofectamine 2000 by following the manufacturer's manual. Forty-eight hours later, cells were harvested, homogenized, and subjected to Western blot analysis of Piezo1 protein expression following the previous protocol [16]. Briefly, the treated HUVECs were homogenized in lysis buffer (1\% SDS, $10 \mathrm{mM}$ Tris $\mathrm{pH} 8.0,130 \mathrm{mM} \mathrm{NaCl}, 5 \mathrm{mM}$ EDTA) containing protease inhibitors. $30 \mu \mathrm{g}$ of total protein was separated on $4-15 \%$ sodium dodecyl sulfate (SDS)-polyacrylamide gel electrophoresis (PAGE) gels and transferred onto polyvinylidene fluoride (PVDF) membranes. After blocking with phosphate buffered saline (PBS) buffer containing $0.1 \%$ Tween-20 (PBST) and 5\% non-fat milk for $30 \mathrm{~min}$, membranes were incubated with rabbit anti-human Piezo1 antibody (Novus Biologicals, Littleton, CO, USA) at room temperature for $2 \mathrm{~h}$, followed by washing with PBST for $3 \times 10 \mathrm{~min}$, and incubation with goat-anti-rabbit IgG antibody (Novus Biologicals) for $2 \mathrm{~h}$ at room temperature. Immunoreactive bands were detected using a chemiluminescent reagent (Pierce, Rockford, IL, USA). To control for loading efficiency, the blots were stripped and re-probed with $\alpha$-tubulin antibody (Cell Signaling Technology, Danvers, MA, USA).

\section{Tube formation assay}

HUVECs in 10-cm dishes were transfected with $10 \mathrm{mM}$ of miR-103a, or cel-miR-39 by lipofectamine 2000. Forty-eight hours later, cells were trypsinized and resuspended in cultured medium containing $1.5 \%$ fetal bovine serum. The tube formation assay was conducted in 24 -well plates coated with $200 \mu \mathrm{L}$ of ECMatrix ${ }^{\mathrm{TM}}$ (Millipore, Billerica, MA, USA) by following the manufacturer's instructions. Cells $\left(1 \times 10^{5}\right.$ cells/well $)$ were then plated onto matrigelcoated plates and incubated at $37^{\circ} \mathrm{C}, 5 \% \mathrm{CO}_{2}$ for $12 \mathrm{~h}$ to allow tube formation and tube formation was evaluated under a light microscope.

\section{Cell viability assay}

$1 \times 10^{4}$ HUVECs were seeded into each well of a 96-well plate. Cells were transfected with $10 \mathrm{mM}$ of miR-103a or cel-miR-39 by lipofectamine 2000 . Forty-eight hours later, the 3-(4,5-dimethylthiazol- 
Table 1. Clinical characteristics of patients.

\begin{tabular}{lcccc}
\hline Characteristics & $\begin{array}{c}\text { AMI }+ \text { HBP } \\
(\mathbf{n}=\mathbf{4 3})\end{array}$ & $\begin{array}{c}\text { AMI } \\
(\mathbf{n}=38)\end{array}$ & $\begin{array}{c}\text { HBP } \\
(\mathbf{n}=45)\end{array}$ & $\begin{array}{c}\text { HC } \\
(\mathbf{n}=47)\end{array}$ \\
\hline Age $[$ years] & $56 \pm 12$ & $55 \pm 11.4$ & $57 \pm 11.5$ & $50 \pm 10.1$ \\
Male/female & $30 / 13$ & $26 / 12$ & $27 / 18$ & $28 / 19$ \\
Hyperlipidemia & $5(11.6 \%)$ & $5(13.2 \%)$ & $3(6.7 \%)$ & $1(2.1 \%)$ \\
Fasting glucose $[\mathrm{mmol} / \mathrm{L}]$ & $6.6 \pm 1.82$ & $6.2 \pm 1.33$ & $6.1 \pm 1.9$ & $5.7 \pm 1.7$ \\
SBP $[\mathrm{mm} \mathrm{Hg}]$ & $143 \pm 17^{*}$ & $121 \pm 14$ & $149 \pm 18^{*}$ & $121 \pm 16$ \\
DBP $[\mathrm{mm} \mathrm{Hg}]$ & $88 \pm 13$ & $80 \pm 14$ & $84 \pm 15$ & $77 \pm 14$ \\
TC $[\mathrm{mmol} / \mathrm{L}]$ & $4.4 \pm 0.9$ & $4.3 \pm 0.8$ & $4.3 \pm 1.0$ & $4.2 \pm 0.8$ \\
TG $[\mathrm{mmol} / \mathrm{L}]$ & $1.41 \pm 1.0$ & $1.42 \pm 0.64$ & $1.58 \pm 1.4$ & $1.6 \pm 1.3$ \\
HDL $[\mathrm{mmol} / \mathrm{L}]$ & $1.04 \pm 0.27$ & $1.05 \pm 0.26$ & $1.22 \pm 0.33$ & $1.26 \pm 0.32$ \\
LDL $[\mathrm{mmol} / \mathrm{L}]$ & $2.71 \pm 0.85$ & $2.66 \pm 0.77$ & $2.5 \pm 0.75$ & $2.4 \pm 0.63$ \\
WBC $\left[\times 10^{9} / \mathrm{L}\right]$ & $8.03 \pm 2.2$ & $8.0 \pm 2.7$ & $6.7 \pm 1.52$ & $6.6 \pm 1.43$ \\
$\mathrm{Cr}[\mu \mathrm{mol} / \mathrm{L}]$ & $98.4 \pm 34$ & $93 \pm 48$ & $68 \pm 27$ & $58 \pm 21$ \\
\hline
\end{tabular}

${ }^{*} \mathrm{p}<0.05$ vs. healthy controls; AMI — acute myocardial infarction; $\mathrm{Cr}$ - creatinine; DBP — diastolic blood pressure; HBP — high blood pressure; HDL - high-density lipoprotein; LDL — low-density lipoprotein; SBP — systolic blood pressure; TC — total cholesterol; TG total triglyceride; WBC — white blood cell

2-yl)-2,5-diphenyltetrazolium bromide (MTT) assay was performed as previously described [17]. The decrease in cell viability was calculated as the percentage of absorbance in the non-transfected control (blank control).

\section{Statistical analysis}

Data were presented as mean \pm standard deviation of the mean (SEM) and analyzed with SPSS 13.0 software. Chi-square statistics $\left(\chi^{2}\right.$ test) were used for analyzing quantitative data. Oneway ANOVA (LSD-t) was used for the comparison among the three groups. A $p<0.05$ was considered statistically significant.

\section{Results}

\section{Clinical and laboratory characteristics}

The general characteristics of patients and healthy controls are presented in Table 1 . No significant differences in age, sex, fasting glucose, total cholesterol, low density lipoprotein, high density lipoprotein, or triglyceride level were observed between patients with HBP and AMI, and patients with AMI and HBP, and the healthy controls. However, significantly higher systolic blood pressure was observed in the two groups of patients with HBP compared to the healthy controls.

\section{The concentration of plasma miRNA-103a}

The concentrations of plasma miR-103a in patients with HBP $(\mathrm{p}<0.05)$, AMI $(\mathrm{p}<0.01)$, and AMI with HBP $(\mathrm{p}<0.001)$ were significantly higher than that in the healthy controls. The concentration of plasma miR-103a in patients with AMI alone was significantly higher than that in patients with HBP alone $(\mathrm{p}<0.05)$, while miR-103a concentrations in patients with AMI and HBP were significantly higher than those in patients with AMI alone $(\mathrm{p}<0.05)$ and patients with HBP alone $(\mathrm{p}<0.01)$ (Fig. 1A). In contrast, the miR-103a expressions in PBMCs were not significantly higher in patients with HBP alone and patients with AMI alone, compared to healthy controls. The miR-103a expression in PBMCs in patients with AMI and HBP was significantly higher than that in the healthy controls $(\mathrm{p}<0.05)$, but not significantly different from that in patients with HBP alone and patients with AMI alone ( $p>0.05)$ (Fig. 1B).

\section{Piezo1 is a target of miR-103a}

A previous study suggested that miR-103a is a mechanosensitive miRNA [9]. The computer informatics assay showed that Piezo1 is a target of miR-103a (Fig. 2A). We further validated whether Piezo1 is a target of miR-103a. Luciferase reporter assay was used to investigate the regulatory effect of miR-103a on Piezo1 expression. The results showed that co-transfection of synthesized miR-103a with pmiR-Piezo1 plasmid, but not pmiR-REPORT blank plasmid significantly decreased luciferase activity in 293T cells (Fig. 2B). To test whether miR-103a regulates Piezo1 expression in HUVECs, cells were transfected with miR-103a, 


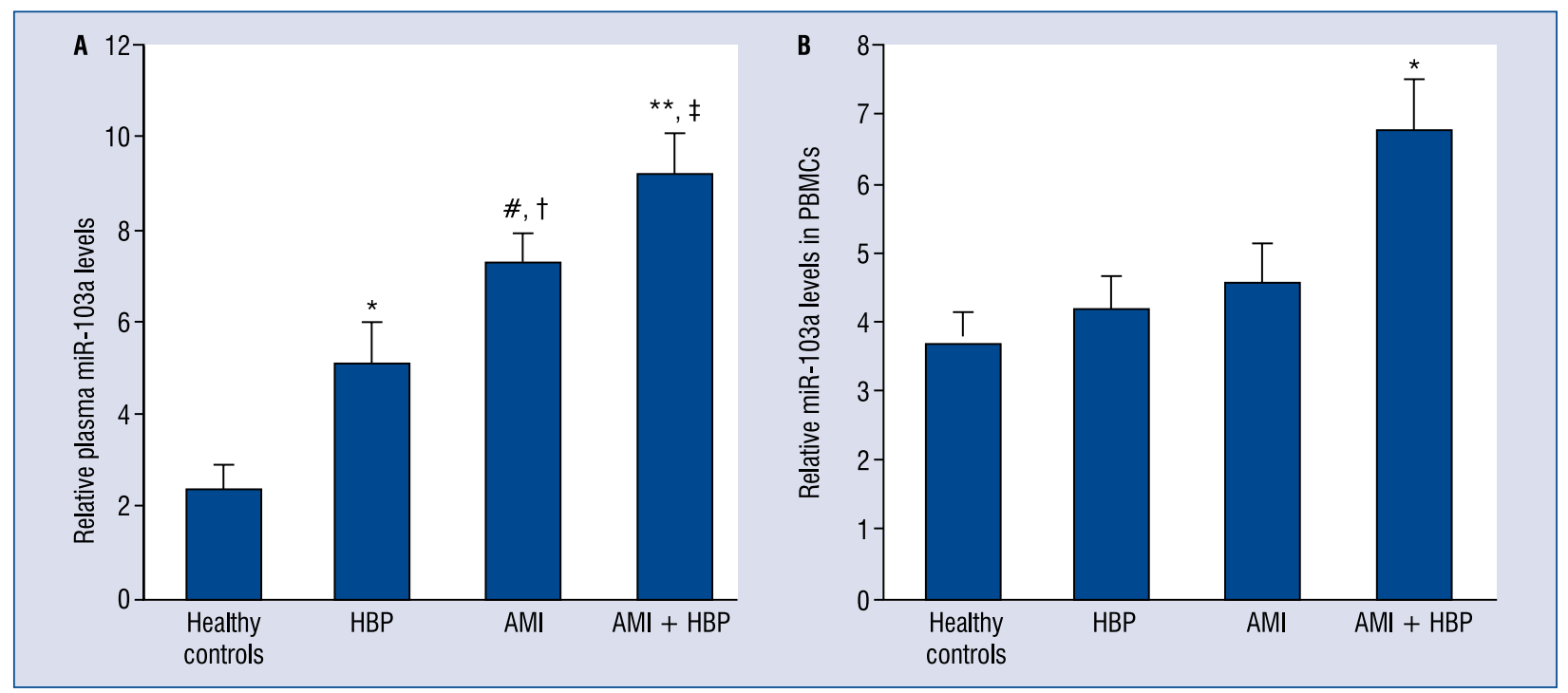

Figure 1. MiR-103a expression in plasma and peripheral blood mononuclear cells (PBMCs). MiR-103a in plasma and PMBCs was measured by real-time polymerase chain reaction and relative expression was calculated; A. MiR-103a expression in plasma, ${ }^{*} p<0.05,{ }^{*} p<0.01,{ }^{* *} p<0.001$ vs. healthy control, ${ }^{\dagger} p<0.05$ vs. high blood pressure (HBP); ${ }^{\ddagger} p<0.05$ vs. acute myocardial infarction (AMI); B. MiR-103a expression in PMBC, ${ }^{*} p<0.05$ vs. healthy control, healthy controls $(n=47)$, HBP $(n=45)$, AMI $(n=38)$, AMI with HBP $(n=43)$.

or cel-miR-39. Western blot showed that HUVECs transfected with miR-103a inhibited Piezo1 protein expression, compared to blank and cel-miR-39 controls (Fig. 2C). These results suggested that Piezo1 is a target of miR-103a in endothelial cells.

\section{Inhibition of capillary tube formation and cell viability in HUVEC by overexpression of miR-103a}

Piezo1 has been demonstrated to regulate the cationic channel activity in endothelial cells [10]. The effect of miR-103a on the ability of HUVEC to form capillary-like structures was tested. As shown in Figure 3A, HUVEC grew on matrigel formed complex network of capillary tubes after $12 \mathrm{~h}$. Transfection with miR-103a significantly inhibited and disrupted capillary tube formation (Fig. 3A, 3B). We further tested effects of miR-103a on cell viability. MTT assay showed that transfection of miR-103a significantly decreased cell viability (Fig. 3C). The transfection efficacy of miR-103a was validated by RT-PCR of miR-103a expression (Fig. 3D).

\section{Discussion}

Previous studies have reported that numerous circulating miRNAs are involved in AMI and some of them are thought to be cardiac-specific
[18-21] and others non-specific [22, 23]. However, previous studies provided no evidence for the pathophysiological functions of these miRNAs in the development of AMI. This study demonstrated that plasma miR-103a is increased in patients with $\mathrm{HBP}$ although it is more significantly increased in AMI patients. The in vitro study further revealed that miR-103a regulated Piezo1 expression and subsequently affected function of endothelial cells. This study suggests that elevated miR-103a might be not only a biomarker for AMI, but also an etiological factor for the development of cardiovascular diseases, including AMI.

In this study, miR-103 was significantly elevated in the plasma of patients with HBP alone. This suggests that elevated miR-103a is associated with HBP and may exist before the onset of AMI. In AMI patients with or without HBP, plasma miR-103a concentrations were more significantly elevated. In contrast, there were no significant differences in miR-103a expressions in peripheral blood mononuclear cells among three patient groups. This implies that peripheral blood cells may not be the source of plasma miR-103a. However, this study also provided no evidence whether the elevated plasma miR-103a came from the necrotic myocardium. In addition, it is difficult to monitor the plasma miR-103a concentration before AMI. Also, blood was collected in AMI patients immedi- 


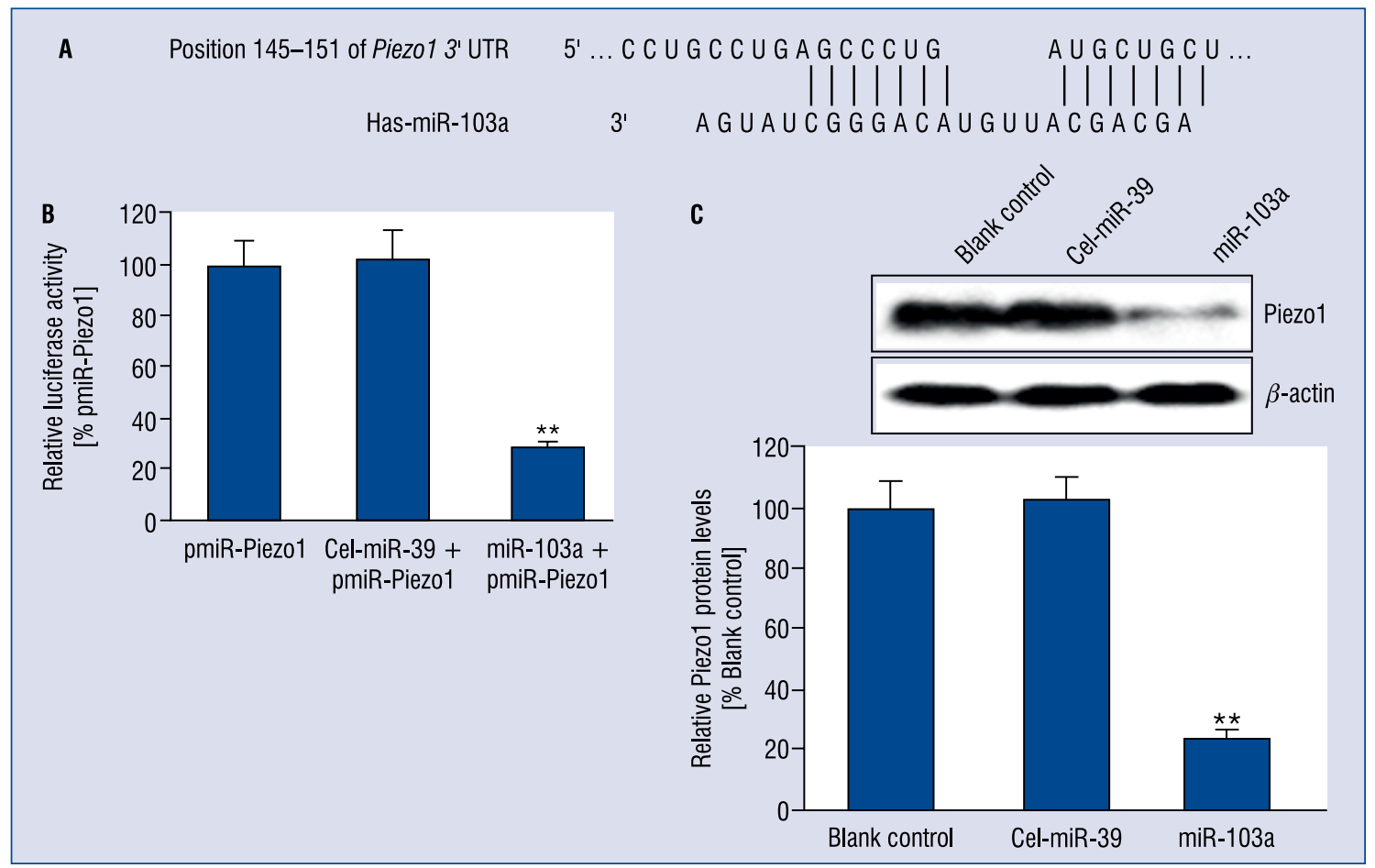

Figure 2. The regulatory effect of miR-103a on Piezo1 expression. A. Bioinformatic analysis of miR-103a targeting site on Piezo1 sequence; B. Luciferase reporting assay. Co-transfection of miR-103a with pmiR-Piezo1 plasmid significantly reduced luciferase activity, $n=5,{ }^{* *} p<0.001$ vs. other groups; C. Western blot of Piezo1 protein expression in human umbilical vein endothelial cells (HUVECs). Transfection of miR-103a obviously decreased Piezo1 protein expression in HUVECs, $n=4,{ }^{* *} p<0.001$ vs. other groups.

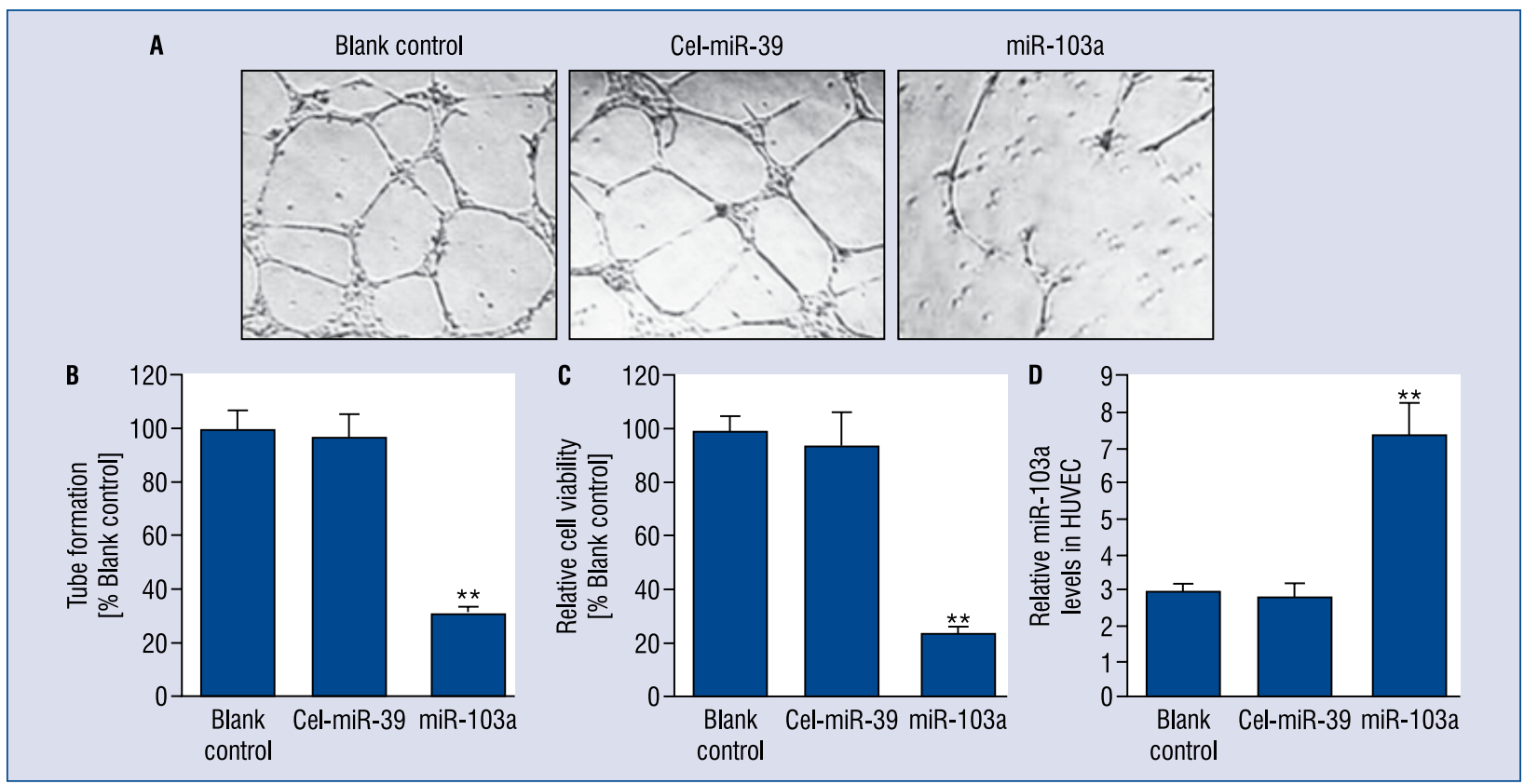

Figure 3. The effects of miR-103a on tube formation and cell viability in human umbilical vein endothelial cells (HUVECs). A. Representative tube formation images. Complex network of tubes were seen in blank and cel-miR-39 controls; B. Quantitative analysis of tube formation. miR-103a transfection significantly decreased tube formation, ${ }^{* *} \mathrm{p}<0.001$ vs. other groups, $\mathrm{n}=5$; C. MTT assay of cell viability. Transfection of miR-103a significantly reduced cell viability in HUVECs, ${ }^{* *} p<0.001$ vs. other groups, $n=4$. D. Real-time polymerase chain reaction of miR-103a expression in HUVECs, ${ }^{* *} p<0.001$ vs. other groups; $n=4$. 
ately at admission, yet fasting blood was collected from HBP patients and healthy controls. It is unclear whether fasting may alter the expressions of miR-103a. Thus, the diagnostic value of elevated plasma miR-103a concentrations in AMI patients may need further evidence because they were only compared to healthy controls, but not with the expression before the onset of AMI.

Piezo1 protein has been identified as an essential component of mechanically activated channels and induces cationic non-selective mechanically activated currents $[24,25]$. The role of Piezo1 gene has been linked recently to the vascular biology [11]. Piezo1 channels have been revealed to be sensors of blood flow through shear-stress-evoked ionic current and calcium influx in endothelial cells, which regulate the spatial reorganization of endothelial cells to the polarity of the applied force [11]. This study revealed that miR-103a directly targeted the Piezo1 gene and inhibited its expression in HUVECs. Artificial overexpression of miR-103a also inhibited capillary tube formation and cell viability in HUVECs. Thus, elevated miR-103 expression in patients with HBP may cause low expression of Piezo1 in endothelial cells and subsequent inhibition of endothelium function. This may be involved in the progression of cardiovascular diseases and the onset of AMI. However, this study provided no evidence on whether elevated miR-103a leads to a decrease in Piezo1 gene expression in the endothelium of patients with HBP and subsequently decreases the sensitivity of endothelium to blood pressure. It is widely known that a single miRNA can target numerous genes. Therefore, besides the Piezo1 gene, it is unclear how many miR-103a-regulated genes are involved in its regulatory effects on the biological functions of endothelial cells. Further studies need to be conducted to clarify the role of miR-103a and Piezo1 in the development of cardiovascular diseases.

\section{Conclusions}

In conclusion, this study suggests that miR-103a might be an etiological factor of HBP through regulating Piezo1 gene expression. Mir-103a could be a biomarker for cardiovascular diseases, including HBP and AMI.

\section{Conflict of interest: None declared}

\section{References}

1. He J, Gu D, Wu X et al. Major causes of death among men and women in China. N Engl J Med, 2005; 353: 1124-1134.
2. Wang H, Eitzman DT. Acute myocardial infarction leads to acceleration of atherosclerosis. Atherosclerosis, 2013; 229: 18-22.

3. Ambros V. The functions of animal microRNAs. Nature, 2004; 431: 350-355.

4. $\mathrm{Xu} \mathrm{W,} \mathrm{San} \mathrm{Lucas} \mathrm{A,} \mathrm{Wang} \mathrm{Z,} \mathrm{Liu} \mathrm{Y.} \mathrm{Identifying} \mathrm{microRNA} \mathrm{targets} \mathrm{in}$ different gene regions. BMC Bioinformatics, 2014; 15 (suppl. 7): S4.

5. Ward J, Kanchagar C, Veksler-Lublinsky I et al. Circulating microRNA profiles in human patients with acetaminophen hepatotoxicity or ischemic hepatitis. Proc Natl Acad Sci USA, 2014; 111: 12169-12174.

6. Boon RA, Dimmeler S. MicroRNAs in myocardial infarction. Nat Rev Cardiol, 2015; 12: 135-142.

7. Long G, Wang F, Duan Q et al. Circulating miR-30a, miR-195 and let-7b associated with acute myocardial infarction. PLoS One, 2012; 7: e50926.

8. Wang F, Long G, Zhao C et al. Atherosclerosis-related circulating miRNAs as novel and sensitive predictors for acute myocardial infarction. PLoS One, 2014; 9: e105734.

9. Zuo B, Zhu J, Li J et al. microRNA-103a Functions as a Mechanosensitive microRNA to Inhibit Bone Formation Through Targeting Runx2. J Bone Miner Res, 2015; 30: 330-345.

10. Li J, Hou B, Beech DJ. Endothelial Piezo1: Life depends on it. Channels (Austin), 2015. [Epub ahead of print].

11. Li J, Hou B, Tumova S et al. Piezo1 integration of vascular architecture with physiological force. Nature, 2014; 515: 279-282.

12. Thygesen K, Alpert JS, Jaffe AS et al. Third universal definition of myocardial infarction. Circulation, 2012; 126: 2020-2035.

13. De Almeida MC, Silva AC, Barral A, Barral Netto M. A simple method for human peripheral blood monocyte siolation. Mem Inst Oswaldo Cruz, 2000; 95: 221-223.

14. Zhang Y, Zhu X, Bai M, Zhang L, Xue L, Yi J. Maternal deprivation enhances behavioral vulnerability to stress associated with miR-504 expression in nucleus accumbens of rats. PLoS One, 2013; 8: e69934.

15. Sourvinou IS, Markou A, Lianidou ES. Quantification of circulating miRNAs in plasma: Effect of preanalytical and analytical parameters on their isolation and stability. J Mol Diagn, 2013; 15: 827-834.

16. Shi Y, Hogue J, Dixit D, Koh J, Olson, Jr. JA. Functional and genetic studies of isolated cells from parathyroid tumors reveal the complex pathogenesis of parathyroid neoplasia. Proc Natl Acad Sci USA, 2014; 111: 3092-3097.

17. Zhang X, Kon T, Wang H et al. Enhancement of hypoxia-induced tumor cell death in vitro and radiation therapy in vivo by use of small interfering RNA targeted to hypoxia-inducible factor1alpha. Cancer Res, 2004; 64: 8139-8142.

18. Adachi T, Nakanishi M, Otsuka Y et al. Plasma microRNA 499 as a biomarker of acute myocardial infarction. Clin Chem, 2010; 56: 1183-1185.

19. Wang GK, Zhu JQ, Zhang JT et al. Circulating microRNA: A novel potential biomarker for early diagnosis of acute myocardial infarction in humans. Eur Heart J, 2010; 31: 659-666.

20. Kuwabara Y, Ono K, Horie T et al. Increased microRNA-1 and microRNA-133a levels in serum of patients with cardiovascular disease indicate myocardial damage. Circ Cardiovasc Genet, 2011; 4: 446-454.

21. Wang F, Long G, Zhao C et al. Plasma microRNA-133a is a new marker for both acute myocardial infarction and underlying coronary artery stenosis. J Transl Med, 2013; 11: 222.

22. Long G, Wang F, Duan Q et al. Human circulating microRNA-1 and microRNA-126 as potential novel indicators for acute myocardial infarction. Int J Biol Sci, 2012; 8: 811-818.

23. Oerlemans MI, Mosterd A, Dekker MS et al. Early assessment of acute coronary syndromes in the emergency department: The potential diagnostic value of circulating microRNAs. EMBO Mol Med, 2012; 4: 1176-1185.

24. Volkers L, Mechioukhi Y, Coste B. Piezo channels: from structure to function. Pflugers Arch, 2015; 467: 95-99.

25. Ranade SS, Qiu Z, Woo SH et al. Piezo1, a mechanically activated ion channel, is required for vascular development in mice. Proc Natl Acad Sci USA, 2014; 111: 10347-10352. 